\title{
Investigation of Motion Picture Processing by Two-Layer Cellular Neural Networks Switching Coupling Templates
}

\author{
Kazushige Natsuno, Yoshihiro Kato, Yoko Uwate and Yoshifumi Nishio \\ Dept. of Electrical and Electronic Engineering, Tokushima University \\ 2-1 Minami-Josanjima,Tokushima, 770-8506, Japan \\ Phone;+81-88-656-7470, FAX:+81-88-656-7471 \\ E-mail: natsushige, kkato, uwate, nishio@ee.tokushima-u.ac.jp
}

\begin{abstract}
In this paper, we propose the new system of two-layer CNN and investigate the output characteristics. In particular, the proposed system is connected to first-layer and second-layer with one coupling template. Also coupling template is switched by each layer of input and output values. In this study, we investigate the output characteristics for changing input images.
\end{abstract}

\section{Introduction}

Our society is called advanced information society. Because of this, the large size of data cause trouble over processing speed. Conventional digital computation methods have problem of processing speed. However analog computation methods are high speed processing. We focus on new computation model of neural networks. The concept of neural networks is neurobiology and adapted to integrated circuits. The key features of neural networks are asynchronous parallel processing, continuous-time dynamics and global interaction of network elements. Cellular Neural Networks (CNN) were introduced by Chua and Yang [1]. CNN is a type of mutual coupling neural network. $\mathrm{CNN}$ is used the concept of cellular automata [2]. The structure of $\mathrm{CNN}$ is grating coupling of circuit. The circuit is called a cell that has an effect on each other. The structure of cell circuit is simple analog circuit. The cell circuit is constructed from linear capacitor, linear resistors, independent voltage source, linear and nonlinear controlled sources. Two-layer $\mathrm{CNN}$ is constructed two conventional single-layer CNNs. The two-layer CNN has two coupling templates. In two single-layer $\mathrm{CNN}$, both layers are effected on each other by two coupling templates. In some image processing, the two-layer CNN is better than the single-layer $\mathrm{CNN}$ [3]. Also, $\mathrm{CNN}$ with switching template is better than the single-layer CNN [4].

In the motion picture processing, a lot of images were continuously processed with time. We consider a new system which the system be processed motion picture by using the input image and previous output image of other layer. In this study, we propose the two-layer CNN with switching only coupling template. The proposed system has only effect from the second-layer to the first-layer. The first-layer and the second-layer are connected by switching templates.

From simulation results, we confirm the effective motion picture processing of the proposed system.

\section{Cellular Neural Network}

In this section, we explain about the single-layer CNN and the two-layer CNN.

\subsection{Single-layer CNN}

In this subsection, we explain the basic structure of the CNN. A block diagram of the conventional single-layer CNN shown Fig. 1. The conventional single-layer CNN use two templates $B$ and $A$ in processing. Template $B$ is used in processing of input image. Template of $A$ is used in processing of feedback of output image.

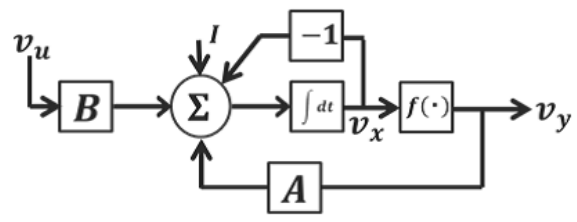

Figure 1: Block diagram of the single-layer CNN.

The state equation and output equation are described as follows.

State equation of the conventional single-layer CNN :

$$
\begin{gathered}
\frac{d v_{x i j}}{d t}=-v_{x i j}+\sum_{k=i-r}^{i+r} \sum_{l=j-r}^{j+r} A_{(i, j ; k, l)} v_{x k l}(t) \\
+\sum_{k=i-r}^{i+r} \sum_{l=j-r}^{j+r} B_{(i, j ; k, l)} v_{u k l}(t)+I \\
(|i-k| \leq 1,|j-l| \leq 1) .
\end{gathered}
$$

Output equation of the conventional single-layer CNN :

$$
v_{y i j}(t)=\frac{1}{2}\left(\left|v_{x i j}(t)+1\right|-\left|v_{x i j}(t)-1\right|\right) .
$$




\subsection{Two-layer CNN}

Figure 2 shows the block diagram of the conventional twolayer $\mathrm{CNN}$. The conventional two-layer $\mathrm{CNN}$ is constructed two conventional single-layers $\mathrm{CNN}$ by coupling two templates $C_{1}$ and $C_{2}$. The coupling templates are used to transferred data between both layers. In addition, the conventional two-layer CNN has been confirmed to have more efficient structure for high performance image processing.

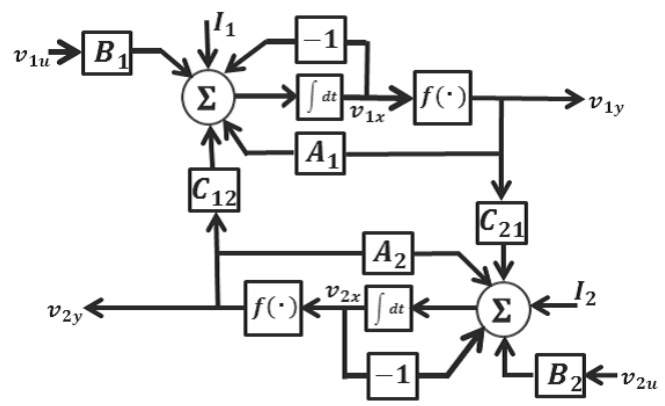

Figure 2: Block diagram of the two-layer CNN.

The state equations and output equations are described as follows.

State equation of second-layer CNN :

$$
\begin{aligned}
& \frac{d v_{1} x i j}{d t}=-v_{1 x i j}+\sum_{k=i-r}^{i+r} \sum_{l=j-r}^{j+r} A_{1(i, j ; k, l)} v_{1 x k l}(t) \\
&+\sum_{k=i-r}^{i+r} \sum_{l=j-r}^{j+r} B_{1(i, j ; k, l)} v_{1 u k l}(t) \\
&+\sum_{k=i-r}^{i+r} \sum_{l=j-r}^{j+r} C_{12(i, j ; k, l)} v_{2 y k l}(t)+I_{1} \\
&(|i-k| \leq 1,|j-l| \leq 1) .
\end{aligned}
$$

Output equation of first-layer CNN :

$$
v_{1} y i j(t)=\frac{1}{2}\left(\left|v_{1} x i j(t)+1\right|-\left|v_{1} x i j(t)-1\right|\right) .
$$

State equation of second-layer $C N N$ :

$$
\begin{aligned}
\frac{d v_{2} x i j}{d t}= & -v_{2 x i j}+\sum_{k=i-r}^{i+r} \sum_{l=j-r}^{j+r} A_{2(i, j ; k, l)} v_{2 x k l}(t) \\
& +\sum_{k=i-r}^{i+r} \sum_{l=j-r}^{j+r} B_{2(i, j ; k, l)} v_{2 u k l}(t) \\
& +\sum_{k=i-r}^{i+r} \sum_{l=j-r}^{j+r} C_{21(i, j ; k, l)} v_{1 y k l}(t)+I_{2} \\
& (|i-k| \leq 1,|j-l| \leq 1) .
\end{aligned}
$$

Output equation of second-layer CNN :

$v_{2} y i j(t)=\frac{1}{2}\left(\left|v_{2} x i j(t)+1\right|-\left|v_{2} x i j(t)-1\right|\right)$.

\section{Proposed Two-layer CNN}

In this section, we explain the system of the proposed twolayer CNN. Figure 3 shows the block diagram of the proposed system. The structure of the proposed system is based on the conventional two-layer CNN. The feature of proposed system is the switching only coupling template. The coupling template is switched two types. The only coupling template is decided by the input values of the first-layer and the output values of the second-layer. The input value of each layer are four type. Input values are switched every $10[\tau]$. After $10[\tau]$, previous output value of first-layer CNN are inputed second-layer.

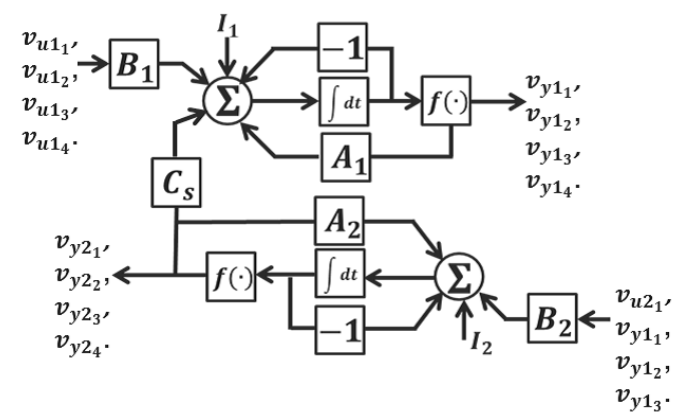

Figure 3: Block diagram of the proposed two-layer CNN.

The algorithm of the proposed system is shown as follows.

Step 1: We determine the difference value $D_{i j}$ by the output value $v_{2 y i j}$ of second-layer and the input value $v_{1 u i j}$ of firstlayer. The difference value is calculated by

$$
D_{i j}=v_{2 y i j}-v_{1 u i j} \text {. }
$$

Step 2: The boundary value and the difference value are compared. If $D_{i j}$ is not 0 , the template of $C_{1}$ is used. On the other hand, if $D_{i j}$ is 0 , the template of $C_{2}$ is used. The switching rule of coupling template is shown as follows.

Switching rule of coupling template :

$$
\begin{aligned}
& D_{i j} \neq 0: C_{1} \text { template }, \\
& D_{i j}=0: C_{2} \text { template. }
\end{aligned}
$$

Step 3 : The value of each cell in the first-layer CNN is updated. The state equation and the output equation of firstlayer are described as follows.

State equation of first-layer CNN :

$$
\begin{aligned}
\frac{d v_{1_{a}} x i j}{d t}= & -v_{1_{a} x i j}+\sum_{k=i-r}^{i+r} \sum_{l=j-r}^{j+r} A_{1(i, j ; k, l)} v_{1_{a} y k l}(t) \\
& +\sum_{k=i-r}^{i+r} \sum_{l=j-r}^{j+r} B_{1(i, j ; k, l)} v_{1_{a} u k l}(t) \\
& +\sum_{k=i-r}^{i+r} \sum_{l=j-r}^{j+r} C_{S}(i, j ; k, l) v_{2_{a} y k l}(t)+I_{1} \\
& (|i-k| \leq 1,|j-l| \leq 1), \\
& (a=1,2,3,4) .
\end{aligned}
$$


Output equation of first-layer CNN :

$$
\begin{aligned}
v_{1_{a}} y i j(t)= & \frac{1}{2}\left(\left|v_{1_{a}} x i j(t)+1\right|-\left|v_{1_{a} x i j}(t)-1\right|\right) \\
& (a=1,2,3,4),
\end{aligned}
$$

where, $a$ means number of input and output images.

Step 4: The value of each cell in the second-layer CNN is updated. Until $10[\tau]$ the state and the output equations are described Eqs. (11) and (12), respectively. After $10[\tau]$ the state and the output equations are described Eqs. (13) and (14), respectively.

State equation of second-layer CNN (until $10[\tau])$ :

$$
\begin{aligned}
\frac{d v_{2_{1}} x i j}{d t}= & -v_{2_{1} x i j}+\sum_{k=i-r}^{i+r} \sum_{l=j-r}^{j+r} A_{2(i, j ; k, l)} v_{2_{1} y k l}(t) \\
& +\sum_{k=i-r}^{i+r} \sum_{l=j-r}^{j+r} B_{2(i, j ; k, l)} v_{2_{1} u k l}(t)+I_{2} \\
& (|i-k| \leq 1,|j-l| \leq 1) .
\end{aligned}
$$

Output equation of second-layer CNN (until $10[\tau])$ :

$v_{2_{1}} y i j(t)=\frac{1}{2}\left(\left|v_{2_{1}} x i j(t)+1\right|-\left|v_{2_{1}} x i j(t)-1\right|\right)$.

State equation of second-layer CNN (after $10[\tau])$ :

$$
\begin{aligned}
\frac{d v_{2} x i j}{d t}= & -v_{2_{2} x i j}+\sum_{k=i-r}^{i+r} \sum_{l=j-r}^{j+r} A_{2(i, j ; k, l)} v_{2 y k l}(t) \\
& +\sum_{k=i-r}^{i+r} \sum_{l=j-r}^{j+r} B_{2(i, j ; k, l)} v_{1 y k l}(t)+I_{2} \\
& (|i-k| \leq 1,|j-l| \leq 1) .
\end{aligned}
$$

Output equation of second-layer CNN (after $10[\tau])$ :

$v_{2_{1}} y i j(t)=\frac{1}{2}\left(\left|v_{2_{1}} x i j(t)+1\right|-\left|v_{2_{1}} x i j(t)-1\right|\right)$.

Step 5 : Step 1 to Step 4 are repeated every $0.005[\tau]$.

\section{Simulation Results}

In this section, we show simulation results of the motion picture processing by using the proposed system. In this simulation, we use the " $3 \times 3$ inverse halftoning" template, which is found in [5]. Templates $A, B$, threshold $I_{1}$ and $I_{2}$ of each layer are assigned as follows.

The template of proposed system :

$$
\begin{aligned}
A_{1} & =A_{2}=0, \\
B_{1} & =B_{2}=\left[\begin{array}{lll}
0.07 & 0.1 & 0.07 \\
0.1 & 0.32 & 0.1 \\
0.07 & 0.1 & 0.07
\end{array}\right], \\
I_{1} & =I_{2}=0 .
\end{aligned}
$$

Using two type of coupling templates are described as follows.

Coupling templates:

$$
\begin{aligned}
C_{1} & =\left[\begin{array}{lll}
0.1 & 0.1 & 0.1 \\
0.1 & -1.5 & 0.1 \\
0.1 & 0.1 & 0.1
\end{array}\right], \\
C_{2} & =\left[\begin{array}{lll}
0.07 & 0.1 & 0.07 \\
0.1 & 0.32 & 0.1 \\
0.07 & 0.1 & 0.07
\end{array}\right] .
\end{aligned}
$$

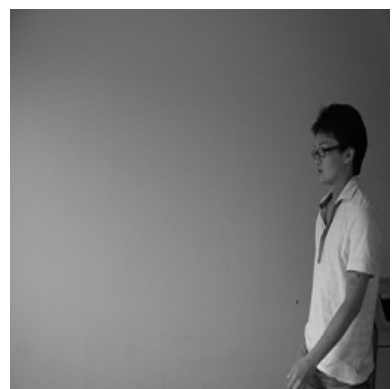

(a)

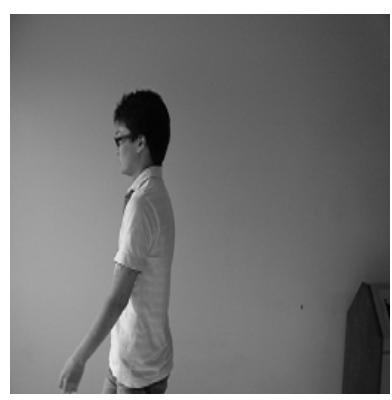

(c)

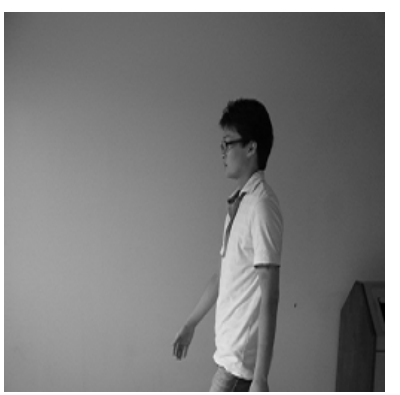

(b)

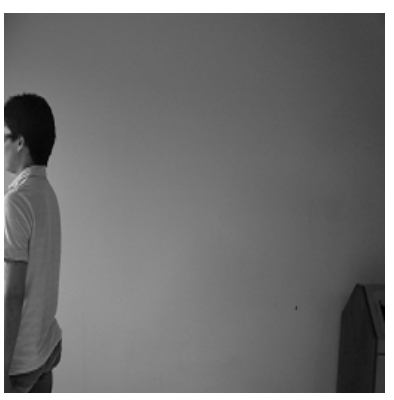

(d)
Figure 4: Input images of first-layer. (a) Input image from 0 [ $\tau]$. (b) Input image from $10[\tau]$. (c) Input image from 20 $[\tau]$. (d) Input image from $30[\tau]$.

In Fig. 4(a), a parson of right side is moving object. In Fig. 4, the parson is moving on to right side from left side. Figure 5 is used first input image of first-layer and initial state images of each layers. The results of the proposed system are shown in Figs. 6 and 7. Figure 6 shows the output images of the first-layer CNN. Figure 7 shows the output images of the second-layer CNN. Figs. 6(b) and 7(c) are similar results. And Figs. 6(c) and 7(d) are similar results. We compare four images in Fig. 6. The moving object of Fig. 6(a) is changed gray-scale value in Fig. 6(b). In Fig. 6(b), moving object of previous output is appeared. Moreover the moving object of previous output has the characteristic of inverse gray-scale values. In comparison of Figs. 6(a) and (c), two previous moving object of Fig. 6(c) is similar gray-scale values of Fig. 6(a). Though, output of Fig. 6(c) is more dilute 


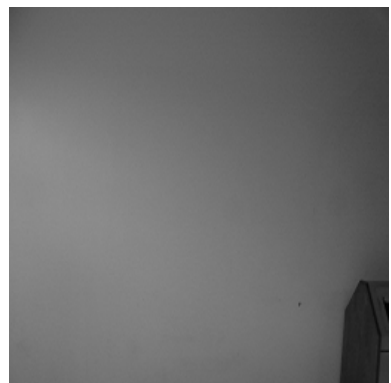

Figure 5: First input image of first-layer and initial state images of each layer.

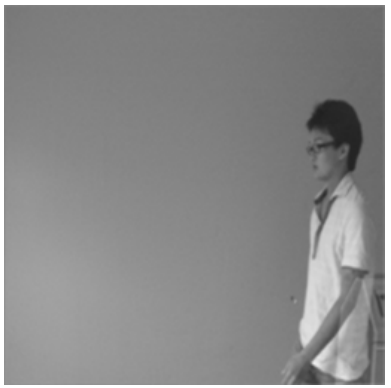

(a)

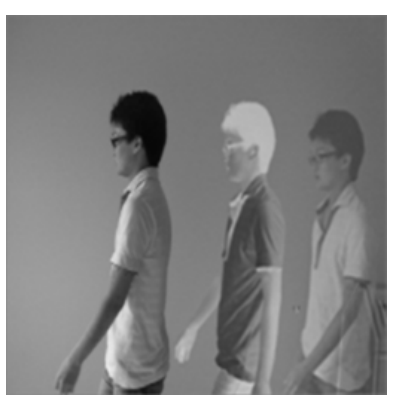

(c)

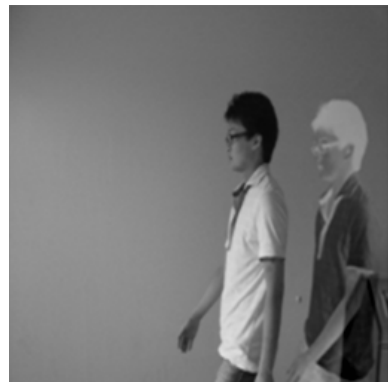

(b)

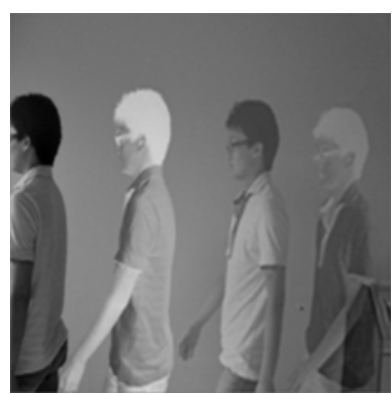

(d)
Figure 6: Output images of first-layer CNN. (a) Output image at $10[\tau]$. (b) Output image at $20[\tau]$. (c) Output image at 30 $[\tau]$. (d) Output image at $40[\tau]$.

than the output of two previous output. Much the same is true on Figs. 6(b) and (d). In addition, in Fig. 6(d), right side object is more dilute than left side object. Therefore, old object is more dilute than new object. The time course of moving object could be discerned by dilute characteristic of output.

From these results, we can say that the proposed system makes it possible to characterize the time course characteristic of moving object.

\section{Conclusion}

In this study, we have proposed two-layer CNN with switching only coupling template. The feature of proposed system is the switching of coupling template. Outputs of second-layer $\mathrm{CNN}$ have an effect on processing of first-layer

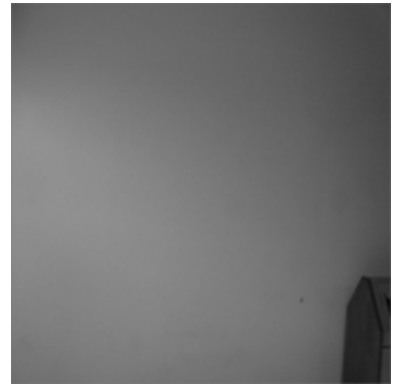

(a)

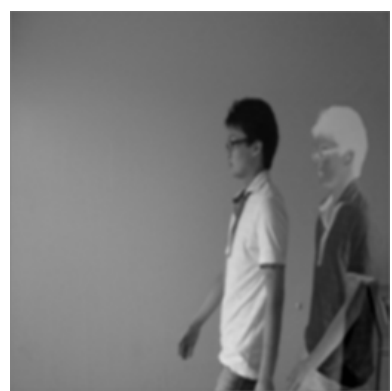

(c)

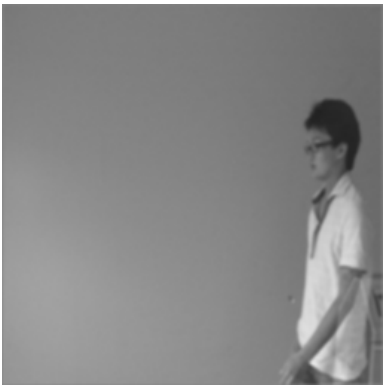

(b)

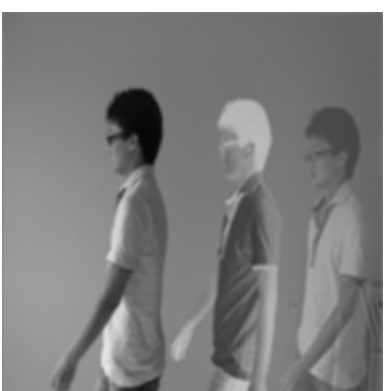

(d)
Figure 7: Output images of second-layer CNN. (a) Output image at $10[\tau]$. (b) Output image at $20[\tau]$. (c) Output image at $30[\tau] .(d)$ Output image at $40[\tau]$.

CNN. The first-layer and the second-layer are connected switching templates. From simulation results, we could say that the proposed system makes it possible to characterize the time course characteristic of moving object.

In the future works, we would like to process the proposed CNN for difficult processing.

\section{Acknowledgment}

This work was partly supported by JSPS Grant-in-Aid for Young Scientists 23700269.

\section{References}

[1] L. O. Chua and L. Yang, "Cellular Neural Networks:Theory," IEEE Trans. Circuits Syst., vol. 32, pp. 1257-1272, Oct. 1988.

[2] S.Wolfram, "Computation theory of cellular automata," Communications in Mathematical Physics, vol. 96, pp.15-57, 1984.

[3] Z. H. Yang, Y. Nishio and A. Ushida, "Image processing of two-layer CNNs applications and stability-," IEICE,Trans. Fundamentals, vol. E85-A, no. 9, pp. 2052-2060, Sept. 2002.

[4] Y. KATO, Y. UEDA, Y. UWATE and Y. NISHIO, "Cellular Neural Networks with Switching Two Types of Templates," Proc. of IJCNN' 11, pp. 14231428, Jun. 2011.

[5] L. Kek, K.Karacs and T .Roska, "Cellular Wave Computing Library(Templates, Algorithms, and Programs), Version 2.1, "Cellular Sensory Wave Computers laboratory, Hungarian Academy of Sciencesk, Budapest, Hungary, 2007. 